\title{
THE ANGULAR MOMENTA OF NEUTRON STARS AND BLACK HOLES AS A WINDOW ON SUPERNOVAE
}

\author{
J. M. Miller ${ }^{1}$, M. C. Miller ${ }^{2}$, And C. S. Reynolds ${ }^{2}$ \\ ${ }^{1}$ Department of Astronomy, University of Michigan, 500 Church Street, Ann Arbor, MI 48109-1042, USA; jonmm@umich.edu \\ ${ }^{2}$ Department of Astronomy, University of Maryland, College Park, MD 20742, USA \\ Received 2010 December 20; accepted 2011 February 7; published 2011 March 17
}

\begin{abstract}
It is now clear that a subset of supernovae displays evidence for jets and is observed as gamma-ray bursts (GRBs). The angular momentum distribution of massive stellar endpoints provides a rare means of constraining the nature of the central engine in core-collapse explosions. Unlike supermassive black holes, the spin of stellar-mass black holes in X-ray binary systems is little affected by accretion and accurately reflects the spin set at birth. A modest number of stellar-mass black hole angular momenta have now been measured using two independent X-ray spectroscopic techniques. In contrast, rotation-powered pulsars spin down over time, via magnetic braking, but a modest number of natal spin periods have now been estimated. For both canonical and extreme neutron star parameters, statistical tests strongly suggest that the angular momentum distributions of black holes and neutron stars are markedly different. Within the context of prevalent models for core-collapse supernovae, the angular momentum distributions are consistent with black holes typically being produced in GRB-like supernovae with jets and with neutron stars typically being produced in supernovae with too little angular momentum to produce jets via magnetohydrodynamic processes. It is possible that neutron stars are with high spin initially and rapidly spun down shortly after the supernova event, but the available mechanisms may be inconsistent with some observed pulsar properties.
\end{abstract}

Key words: accretion, accretion disks - black hole physics - gamma-ray burst: general - stars: evolution - stars: neutron - supernovae: general

Online-only material: color figure

\section{INTRODUCTION}

The angular momentum or spin period of a rotating star is easily defined and understood. To make a consistent comparison with black holes, however, the dimensionless angular momentum must be used. This quantity is referred to as the "spin parameter" or "spin" of a black hole, and it is given by $a=c J / G M^{2}$, where $-1 \leqslant a \leqslant 1$ (where $J$ is the angular momentum, $G$ is Newton's gravitational constant, and $M$ is the mass of the black hole). Interactions between the disk and the hole likely enforce a limit of $a \leqslant 0.998$ (Thorne 1974), assuming zero torque at the innermost disk edge. For likely neutron star radii and masses, rotation at the break-up frequency would give a spin parameter of $a \simeq 0.7$.

Efforts to measure and constrain cosmic acceleration, for instance, are aided by the ability to rely on samples of sources rather than individual measurements and by the fact of independent measurement techniques. The case is similar in the young enterprise of black hole spin measurement: independent measurement techniques are being exploited and small samples have been compiled. Modeling thermal continuum emission from the accretion disk provides one means of measuring black hole spin (see, e.g., Shafee et al. 2006; McClintock et al. 2006). Modeling the dynamical broadening of emission lines excited in the disk by external irradiation (disk reflection) provides a second, independent means of measuring black hole spin (see, e.g., Miller et al. 2002, 2009; Reis et al. 2009; for a recent review; see Miller 2007). Both methods rely on the premise that the innermost edge of the accretion disk is sensitive to the innermost stable circular orbit (ISCO; see, e.g., Bardeen et al. 1972), which is a complex but monotonic function of the black hole spin parameter.

A black hole with zero spin must accrete half of its initial mass to achieve a spin of $a=0.84$ and considerably more mass to achieve even higher spins (Bardeen 1970). In an X-ray binary, then, accretion does not strongly affect the spin of the hole: a low-mass companion cannot donate enough mass and a high-mass companion cannot donate enough mass in its short lifetime. As a result, the spin of stellar-mass black holes reflects the value imparted at birth. In contrast, the spin of neutron stars can change dramatically. Magnetic braking can act to spin down a neutron star, while accretion in an X-ray binary can act to greatly increase the spin frequency. However, in a small but growing number of isolated, rotation-powered pulsars, it has recently been possible to estimate the natal spin period of the star. This requires a measurement of the magnetic dipole braking index and/or a knowledge of the age of the supernova remnant in which the neutron star was formed.

Detections of supernovae-like spectra in the afterglow of gamma-ray bursts (GRBs) clearly associate long-duration GRBs with a subset of core-collapse supernovae (Bloom et al. 1999; Stanek et al. 2003). The "collapsar" model (Woosley 1993; MacFadyen \& Woosley 1999) is widely accepted as the standard model for long-duration GRBs. The jets predicted in these supernova models are in broad agreement with evidence of beaming (Frail et al. 2001). The spin parameter of stellar endpoints can provide a rare window into the central engine of supernovae and GRBs, and may be important in understanding why a subset of supernovae displays jets. In this work, we exploit the small but growing number of stellar-mass black hole spin measurements and natal neutron star spin periods, and explore potential consequences for prevalent supernova models.

\section{DATA SELECTION}

A set of nine estimated natal spin periods for isolated, rotation-powered pulsars is given in Table 7 of Faucher-Giguere 
Table 1

Black Hole Angular Momenta

\begin{tabular}{lcc}
\hline \hline \multicolumn{1}{c}{ Source } & $\begin{array}{c}c J / G M^{2} \\
(\text { reflection })\end{array}$ & $\begin{array}{c}c J / G M^{2} \\
(\text { continuum) }\end{array}$ \\
\hline M33 X-7 & $\ldots$ & $0.77(5)^{\mathrm{a}}$ \\
LMC X-1 & $\ldots$ & $0.92(6)^{\mathrm{b}}$ \\
A 0620-00 & $\ldots$ & $0.12(19)^{\mathrm{c}}$ \\
4U 1543-475 & $0.3(1)^{\mathrm{d}}$ & $0.80(5)^{\mathrm{e}}$ \\
XTE J1550-564 & $0.76(1)^{\mathrm{d}}$ & $\ldots$ \\
XTE J1650-500 & $0.79(1)^{\mathrm{d}}$ & $\ldots$ \\
XTE J1652-453 & $0.45(2)^{\mathrm{f}}$ & $\ldots$ \\
GRO J1655-40 & $0.98(1)^{\mathrm{d}}$ & $0.70(5)^{\mathrm{e}}$ \\
GX 339-4 & $0.94(2)^{\mathrm{d}}$ & $\ldots$ \\
SAX J1711.6-3808 & $0.6(3)^{\mathrm{d}}$ & $\ldots$ \\
XTE J1752-223 & $0.55(11)^{\mathrm{g}}$ & $\ldots$ \\
Swift J1753.5-0127 & $0.76(13)^{\mathrm{h}}$ & $\ldots$ \\
XTE J1908+094 & $0.75(9)^{\mathrm{d}}$ & $\ldots$ \\
GRS 1915+105 & $0.98(1)^{\mathrm{i}}$ & $\ldots$ \\
Cygnus X-1 & $0.05(1)^{\mathrm{d}}$ & $\ldots$ \\
\hline
\end{tabular}

Notes. Measured values of black hole spin parameters are given above. The errors are statistical errors on the last significant digit. References. ${ }^{\mathrm{a}}$ Liu et al. 2008; ${ }^{\mathrm{b}}$ Gou et al. 2009; ${ }^{\mathrm{c}}$ Gou et al. 2010; d Miller et al. 2009; ' Shafee et al. 2006; ${ }^{\mathrm{f}}$ Hiemstra et al. 2011; ${ }^{\mathrm{g}}$ Reis et al. 2011; ${ }^{\mathrm{h}}$ Reis et al. 2009; ${ }^{\mathrm{i}}$ Blum et al. 2009; ${ }^{\mathrm{j}}$ McClintock et al. 2006.

\& Kaspi (2006). These sources and their natal spin periods are treated in detail in this analysis. In the two cases where an initial period is given as a lower limit, we have conservatively adopted the limiting value (this will give higher dimensionless angular momenta).

The measurement of black hole spins is in its infancy, and so we imposed some modest quality metrics in selecting black hole data. We required that all spin data must be based on statistical fits wherein a trial spectral model was folded through an instrument response and evaluated against an observed spectrum using a goodness-of-fit statistic. Owing to the lack of errors and goodness-of-fit statistics, then, we have excluded early spin estimates by Zhang et al. (1997). Blum et al. (2009) report two spin values for GRS $1915+105$ based on fits to the disk reflection spectrum obtained with Suzaku. The higher value of $a=0.98 \pm 0.01$ is used in this work, as it derives from fits made to a spectrum spanning a much broader energy range. A lower value for the spin of GRS $1915+105$ has also been reported based on fits to the disk continuum (Middleton et al. 2006). The high value listed in Table 1 is likely more robust as it was derived by giving extra weight to observations wherein a standard Novikov \& Thorne (1973) accretion disk is likely to hold (McClintock et al. 2006). A quality cut is automatically imposed on spin measurements made using thermal continuum emission from the accretion disk, in that the mass and distance to a source must be well determined if fits are to yield meaningful constraints.

Table 1 details the black hole dimensionless angular momenta used in this work. In five of the black hole systems listed (XTE J1652-453, SAX J1711.6-3808, XTE J1752-223, Swift J1753.5-0127, and XTE J1908+094), strong dynamical constraints requiring a black hole primary have not yet been obtained through radial velocity techniques. However, myriad phenomena observed from these sources are consistent with dynamically constrained systems, and it is extremely likely that these X-ray binaries harbor black holes (for a review of characteristic properties; see Remillard \& McClintock 2006).
Table 2

Distribution Properties

\begin{tabular}{lll}
\hline \hline \multicolumn{1}{c}{ Sample } & $a_{\text {mean }}$ & $a_{\text {median }}$ \\
\hline BH (reflection) & 0.66 & 0.76 \\
BH (continuum) & 0.72 & 0.80 \\
NS (natal; $\left.1.4 M_{\odot}, R=15 \mathrm{~km}\right)$ & 0.029 & 0.017 \\
NS (natal; $\left.1.4 M_{\odot}, R=10 \mathrm{~km}\right)$ & 0.018 & 0.007 \\
\hline
\end{tabular}

\section{ANALYSIS}

Black hole angular momenta are measured using relativistic spectroscopic models; no additional calculations are required to make use of these data. In the case of neutron stars, it is necessary to calculate the angular momentum of each star using its mass and spin period. If the equation of state of ultra-dense matter was known, the moment of inertia could be calculated by integrating the prescription for how mass varies with radius. At present, the correct equation of state is unknown and many candidates exist (see Lattimer \& Prakash 2006). It is worth noting that some soft equations of state have recently been ruled out with the discovery of an especially massive neutron star $\left(M=1.97 \pm 0.04 M_{\odot}\right.$; Demorest et al. 2010). In order not to bias our analysis in favor of any particular equation of state, we have simply approximated the moment of inertia as $I=(2 / 5) M R^{2}$ as per a sphere of uniform density.

Dimensionless angular momenta were calculated using the natal periods given in Faucher-Giguere \& Kaspi (2006), for different combinations of neutron star mass and radius. One set of dimensionless angular momenta was calculated assuming canonical parameters: $R=10 \mathrm{~km}$ and $M_{\mathrm{NS}}=1.4 M_{\odot}$. Extreme dimensionless angular momenta result from assuming a larger radius for a given mass. All of the equations of state treated in Lattimer \& Prakash (2006) predict radii less than or equal to $15 \mathrm{~km}$. A set of extreme angular momenta was therefore derived assuming $R=15 \mathrm{~km}$ and $M_{\mathrm{NS}}=1.4 M_{\odot}$.

The mean and median values of the dimensionless angular momentum distributions are given in Table 2, and the distributions are shown in Figure 1. The black hole distributions are consistent with moderately high values: $a_{\text {mean }}=0.66$ for spins derived using disk reflection spectra and $a_{\text {mean }}=0.72$ for spins derived using the disk continuum. These values stand in marked contrast to those derived for the neutron star samples. For neutron stars where the natal spin period has been estimated, even extreme stellar parameters only work to yield $a_{\text {mean }}=0.029$.

The sample of black hole spin parameters derived using disk reflection fits is small (12 measurements), but twice as large as the present sample derived using the disk continuum. A Gaussian fit to the reflection-derived distribution gives $a_{\text {cent }}=$ 0.71 and $\sigma=0.26$. A fit to the smaller distribution of continuum-derived spin parameters gives $a_{\text {cent }}=0.81$ and $\sigma=0.06$ (the width should be viewed as a lower limit as the fit was largely insensitive to the lowest spin value). Although likely aided by the small sample sizes currently available, the spin distributions found using these independent methods are formally consistent. The sample of natal neutron star periods is also small, with only nine estimates. Assuming the extreme stellar parameters, a Gaussian fit to this distribution gives $a_{\text {cent }}=2.1 \times 10^{-2}$ and $\sigma=1.9 \times 10^{-2}$. Assuming canonical stellar parameters, a Gaussian fit gives $a_{\text {cent }}=9.5 \times 10^{-3}$ and $\sigma=8.4 \times 10^{-3}$.

Table 3 lists the results of running two-sided KolmogorovSmirnov (K-S) tests on different angular momentum distributions. This statistical test measures the probability that two 


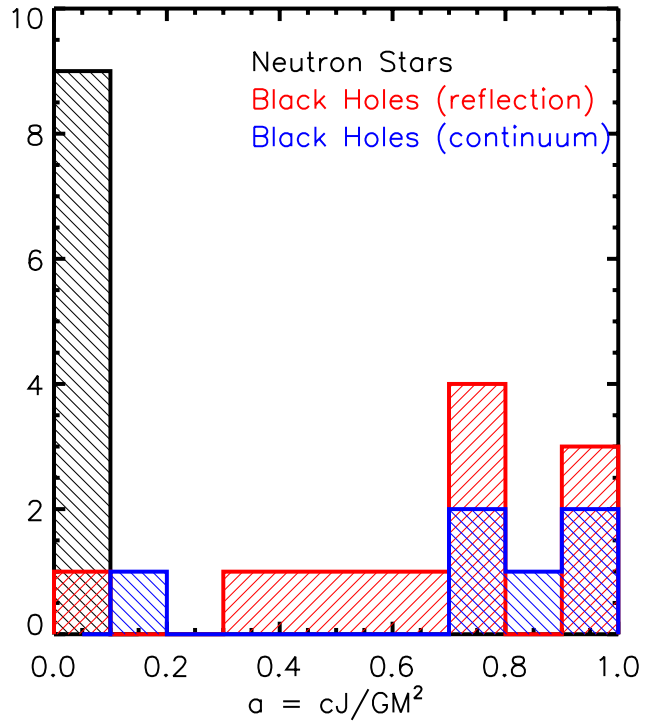

Figure 1. Distribution of dimensionless angular momenta for neutron stars and stellar-mass black holes, compiled from recent measurements, is shown here. The neutron star momenta were calculated using the subset of rotation-powered pulsars wherein natal spin periods have been estimated. Stellar radii of $15 \mathrm{~km}$ and masses of $1.4 M_{\odot}$ were assumed in all cases in order to give the greatest possible angular momentum values. A two-sided Kolmogorov-Smirnov test was used to evaluate the probability that neutron star and black hole spins were drawn from the same parent distribution. A probability of $3.6 \times 10^{-4}$ is found when comparing neutron star spins to black hole spins derived using the disk continuum. The probability is $9.3 \times 10^{-5}$ when using black hole spins derived using disk reflection.

(A color version of this figure is available in the online journal.)

samples are drawn from the same parent distribution. For the samples and assumed stellar parameters considered in this work, the highest probability that the neutron star and black hole distributions are drawn from the same parent is just $3.6 \times 10^{-4}$. Despite the fact that the sample of black hole spin parameters and natal neutron star spin periods is small, it is clear that they belong to distinct distributions. Black holes are likely born with high spin parameters, and neutron stars may be born with very low spin parameters.

At the time of writing, the Australia Telescope National Facility Pulsar Catalog (Manchester et al. 2005) contains 1875 rotation-powered pulsars. The spins of the pulsars in this compilation do not reflect birth conditions. We simply note that no pulsar in this sample has a dimensionless spin parameter in excess of $a=0.3$, neither for typical nor extreme stellar parameters, and the sample is statistically distinct from the black hole distributions at extremely high confidence.

It is difficult to assess the degree to which current black hole spin measurements may be affected by systematic errors and skew comparisons to the sample of neutron star angular momenta. The thermal continuum emission from stellar-mass black hole accretion disks can be fit well with simple, twoparameter models that lack inner torque conditions and provide no direct spin constraint. New models that include spin as a variable parameter are more complex and rely on accurate measures of the mass and distance of the black hole, as well as reasonable estimates of the mass accretion rate and scattering in the disk atmosphere (see, e.g., McClintock et al. 2006; Nowak et al. 2008). Spin constraints derived based on fits to disk reflection spectra have primarily made use of relatively simple models which assume a constant disk density (see, e.g., Miller et al. 2009). A few spectral fits have been made with more
Table 3

Statistical Tests

\begin{tabular}{lcc}
\hline \hline \multicolumn{1}{c}{ Sample } & \multicolumn{1}{c}{ Sample } & K-S Probability \\
\hline BH (reflection) & NS (natal; $\left.1.4 M_{\odot}, 15 \mathrm{~km}\right)$ & $9.3 \times 10^{-5}$ \\
BH (reflection) & NS (natal; $\left.1.4 M_{\odot}, 10 \mathrm{~km}\right)$ & $1.4 \times 10^{-5}$ \\
BH (continuum) & NS (natal; $\left.1.4 M_{\odot}, 15 \mathrm{~km}\right)$ & $3.6 \times 10^{-4}$ \\
BH (continuum) & NS (natal; $\left.1.4 M_{\odot}, 10 \mathrm{~km}\right)$ & $3.6 \times 10^{-4}$ \\
\hline
\end{tabular}

physical reflection models; these fits appear to give consistent results (see, e.g., Reis et al. 2008), but more work is needed.

For three black holes in Table 1, spins have been measured using both techniques, and the values do not agree in detail. All of the spin values and their associated errors were derived based on spectral fitting that minimized a $\chi^{2}$ statistic. The resulting $\chi^{2}$ space can be complex, and using a $1 \sigma$ error to evaluate the degree to which measurements disagree can give an overestimate (see Miller et al. 2009). The measurements made for 4U 1543-475 are most strongly at odds. In this case, the low resolution and calibration of the gas proportional counter spectrum may serve to underestimate the extent of the iron line; a single spectral bin could account for $\Delta(a) \simeq 0.1-0.2$. The low spin value for Cygnus X-1 may be another good example of a suspicious value. It derives from fits to the source in a "low/hard" state, wherein the disk may not always extend to the ISCO (see, e.g., Esin et al. 1997). Measurements of the inner disk radius based on relativistic reflection modeling in states where the disk is more certain to extend to the ISCO give values as small as $r_{i n}=2.2 \pm 1.0 G M / c^{2}$ (Miller et al. 2005), which translates into $a=0.9_{-0.2}^{+0.1}$.

In two cases, then, potential systematic errors resulting from limited spectral resolution and source phenomenology may have served to underestimate the degree to which black hole and neutron star angular momentum distributions differ. An uncertainty in basic accretion disk physics, however, could potentially work in the opposite sense. Both techniques assume that the inner edge of an actual accretion disk is truncated at the test particle ISCO. If real fluid accretion disks push across the ISCO defined by test particle orbits, both spin measurement techniques would give falsely high spin values. New numerical simulations aimed at addressing this specific issue appear to justify the underlying assumption of both techniques (see, e.g., Shafee et al. 2008; Reynolds \& Fabian 2008). However, this must be continually revisited as numerical techniques and computing power advance.

\section{DISCUSSION AND CONCLUSIONS}

The dimensionless angular momenta of stellar-mass black holes and rotation-powered neutron stars are compared statistically. Two different methods of measuring black hole spin parameters are considered, and both canonical and extreme stellar parameters were considered for neutron stars with estimated natal spin periods. A strong statistical difference between the distributions signals that black holes could typically born with dramatically higher dimensionless angular momentum than neutron stars. The observed dichotomy in their dimensionless angular momenta implies that there may be important differences in the core-collapse supernovae events that create these stellar remnants.

The collapse of a $35 M_{\odot}$ star with a $14 M_{\odot}$ He core is examined in detail by MacFadyen \& Woosley (1999). Reasonable pre-collapse angular momentum values for the eventual iron 
core are found to lead to the formation of a disk that rapidly accretes onto a newly formed black hole, quickly giving a spin parameter of $a \simeq 0.9$. Our results are consistent with this prediction (see Tables 1 and 2, and Figure 1), lending support to the overall model described by MacFadyen \& Woosley (1999). Of course, this model is the standard "collapsar" picture for the production of GRBs, and it is interesting to note that the same disk required for driving the new black hole to a high spin parameter is also responsible for producing a jet via magnetohydrodynamic (MHD) processes.

Shibata \& Shapiro (2002) used simulations to understand the spin of the black hole left by a uniformly rotating supermassive star, finding $a \simeq 0.75$. Shapiro \& Shibata (2002) later showed that the same result can be derived analytically (also see Gammie et al. 2004). These treatments are primarily concerned with extremely massive stars as candidate progenitors for the buildup of supermassive black holes in the early universe. Although our results are consistent with these predictions as well, the detailed models of MacFadyen \& Woosley (1999) may be more relevant to the specific case of stellar-mass black holes.

For both canonical and extreme stellar parameters, the sample of natal neutron star spin periods strongly implies that these remnants could be born with very low dimensionless angular momenta. This stands in stark contrast to some theoretical treatments of core-collapse supernovae, which suggest that neutron stars could plausibly be born with spin periods of $1 \mathrm{~ms}$ and $a \simeq 0.7$ (e.g., Heger et al. 2000). A variety of different effects have been proposed to explain how a neutron star might be rapidly spun down to a point that agrees with inferred natal spin periods.

$R$-mode instabilities on the surface of a hot neutron star might lead to significant gravitational wave losses and quickly increase the spin period (Andersson 1998; Andersson et al. 1999). Due to the low saturation amplitude of the instabilities and their strong spin-frequency dependence, however, it may take thousands of years to spin down neutron stars with moderate initial spins (Arras et al. 2003; Heger et al. 2005; Ott et al. 2006). Advances in gravitational wave detection may eventually enable a test of this spin-down mechanism.

Spin-down via the magnetic "propeller" mechanism may be another viable means of quickly increasing the period of neutron stars that are born spinning very rapidly. However, this picture requires significant "fallback" accretion after the initial explosion, and fallback may not be important in the majority of supernovae (Ott et al. 2006).

Spruit \& Phinney (1998) have proposed that neutron star spins might be imparted by off-center kicks rather than angular momentum conservation within a contracting star. In this picture, the progenitor star rotates as a solid body prior to collapse, leaving a very slowly rotating neutron star. Asymmetries in the collapse give an off-center kick to the neutron star, imparting the higher spins that are observed. Thus, pulsar kicks and spin periods should be positively correlated. This is a particularly appealing possibility in that two phenomena are explained through a common and fairly simple process. However, the predicted link between spin period and kick velocity may be at odds with data (see, e.g., Kaspi \& Helfand 2002).

Differential rotation in a massive star may be a viable means of producing the observed distribution of natal neutron star spins and obviate the need for spin-down mechanisms that act shortly after birth. Heger et al. (2005) examine the effects of differential rotation in a $15 M_{\odot}$ star and find that plausible magnetic torques could reduce the rotation rate of the neutrons star by factors of 30-50 compared to scenarios where magnetic fields are unimportant. The specific angular momentum of the pre-collapse core predicted in these magnetic torque models is below the threshold required to produce strong MHD jets in the "collapsar" model of MacFadyen \& Woosley (1999), for enclosed masses less than $3.2 M_{\odot}$, and perhaps even up to $6 M_{\odot}$. This may imply that black holes with small masses could have relatively low spin parameters.

At least within the framework of prevailing models for core-collapse supernovae, and subject to the limitations and systematics of the small, new samples examined, our results are consistent with most black holes being born with relatively high spin in GRB-like supernovae events with jets, while neutron stars (and a small subset of black holes) may be born in corecollapse supernovae wherein some effect-plausibly magnetic torques - has acted to reduce the angular momentum of the stellar core and thus inhibited jet production. It is unclear why magnetic torques, fallback, or other effects may be manifest in some core-collapse events, but not others.

As the number of black hole spin measurements and natal neutron star spin periods increase over time, and as the field matures, the results of this early analysis may change to a degree. A more enduring result may simply be that we are now able to utilize black hole angular momenta, natal neutron star angular momenta, and comparisons between the two as tools to better understand core-collapse supernovae and massive stellar evolution.

We thank Charles Gammie, Andy Fabian, Victoria Kaspi, and Stuart Shapiro for helpful discussions. We thank the referee, David Helfand, for a careful review that improved this manuscript. M.C.M. acknowledges support through NSF grant AST0708424. C.S.R. acknowledges NASA support under grant NNX09AC09G.

\section{REFERENCES}

Andersson, N. 1998, ApJ, 502, 708

Andersson, N., Kokkotas, K., \& Schutz, B. F. 1999, ApJ, 510, 846

Arras, P., Flanagan, E. E., Morsink, S. M., Schenk, A. K., Teukolsky, S. A., \& Wasserman, I. 2003, ApJ, 591, 1129

Bardeen, J. M. 1970, Nature, 226, 64

Bardeen, J. M., Press, W. H., \& Teukolsky, S. A. 1972, ApJ, 178, 347

Bloom, J. S., et al. 1999, Nature, 401, 453

Blum, J. L., et al. 2009, ApJ, 706, 60

Demorest, P. B., Pennucci, T., Ransom, S. M., Roberts, M. S. E., \& Hessels, J. W. T. 2010, Nature, 467, 1081

Esin, A., McClintock, J., \& Narayan, R. 1997, ApJ, 489, 865

Faucher-Giguere, C.-A., \& Kaspi, V. M. 2006, ApJ, 643, 332

Frail, D. A., et al. 2001, ApJ, 562, L55

Gammie, C. F., Shapiro, S. L., \& McKinney, J. C. 2004, ApJ, 602, 312

Gou, L., McClintock, J. E., Steiner, J. F., Narayan, R., Cantrell, A. G., Bailyn, C. D., \& Orosz, J. A. 2010, ApJ, 718, L122

Gou, L., et al. 2009, ApJ, 701, 1076

Heger, A., Langer, N., \& Woosley, S. E. 2000, ApJ, 528, 368

Heger, A., Woosley, S. E., \& Spruit, H. C. 2005, ApJ, 626, 350

Hiemstra, B., Méndez, M., Done, C., Díaz Trigo, M., Altamirano, D., \& Casella, P. 2011, MNRAS, 411, 137

Kaspi, V. M., \& Helfand, D. J. 2002, in ASP Conf. Ser. 271, Neutron Stars in Supernova Remnants, ed. P. O. Slane \& B. M. Gaensler (San Francisco, CA: ASP), 3

Lattimer, J. M., \& Prakash, M. 2006, Nucl. Phys. A, 777, 479

Liu, J., et al. 2008, ApJ, 679, L37

MacFadyen, A. I., \& Woosley, S. E. 1999, ApJ, 524, 262 
Manchester, R. N., Hobbs, G. B., Teoh, A., \& Hobbs, M. 2005, AJ, 129 1993

McClintock, J. E., et al. 2006, ApJ, 652, 518

Middleton, M., Done, C., Gierlinski, M., \& Davis, S. W. 2006, MNRAS, 373, 1004

Miller, J. M. 2007, ARA\&A, 45, 441

Miller, J. M., Fabian, A. C., Nowak, M. A., \& Lewin, W. H. G. 2005, in Proc. the 10th Marcel Grossmann Meeting, Rio de Janiero Brazil, 2003, ed. M Novello, S. P. Berliaffa, \& R. Ruffini (Singapore: World Scientific), 1296

Miller, J. M., et al. 2002, ApJ, 570, L69

Miller, J. M., et al. 2009, ApJ, 697, 900

Novikov, I., \& Thorne, K. S. 1973, in Black Holes, ed. C. De Witt \& B. De Witt (New York: Gordon and Breach), 343

Nowak, M. A., Juett, A., Homan, J., Yao, Y., Wilms, J., Schulz, N. S., \& Canizares, C. R. 2008, ApJ, 689, 1199

Ott, C. D., Burrows, A., Thompson, T. A., Livne, E., \& Walder, R. 2006, ApJ, 164,130
Reis, R. C., Fabian, A. C., Ross, R. R., \& Miller, J. M. 2009, MNRAS, 395, 1257

Reis, R. C., Fabian, A. C., Ross, R. R., Miniutti, G., Miller, J. M., \& Reynolds, C. 2008, MNRAS, 387, 1489

Reis, R. C., et al. 2011, MNRAS, 410, 2497

Remillard, R. A., \& McClintock, J. E. 2006, ARA\&A, 44, 49

Reynolds, C. S., \& Fabian, A. C. 2008, ApJ, 675, 1048

Shafee, R., McKinney, J. C., Narayan, R., Tchekhovskoy, A., Gammie, C. F., \& McClintock, J. E. 2008, ApJ, 687, L25

Shafee, R., et al. 2006, ApJ, 636, L113

Shapiro, S. L., \& Shibata, M. 2002, ApJ, 577, 904

Shibata, M., \& Shapiro, S. L. 2002, ApJ, 572, L39

Spruit, H., \& Phinney, E. S. 1998, Nature, 393, 139

Stanek, K. Z., et al. 2003, ApJ, 591, L17

Thorne, K. S. 1974, ApJ, 191, 507

Woosley, S. 1993, ApJ, 405, 273

Zhang, S. N., Cui, W., \& Chen, W. 1997, ApJ, 482, L155 